MS. GRACE MORRIS (Orcid ID : 0000-0003-3598-9712)

DR. TIM OUTHRED (Orcid ID : 0000-0003-2534-3635)

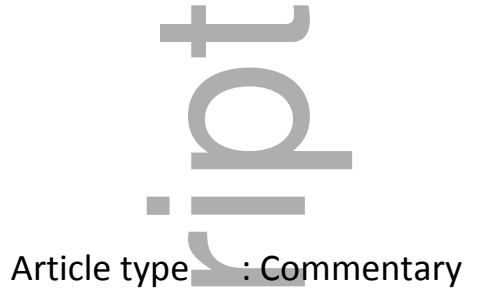

Commentary
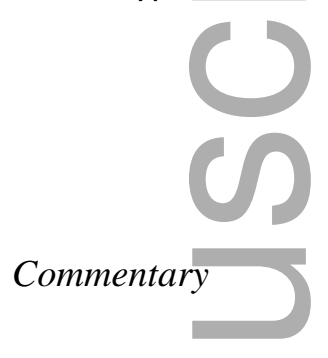

\title{
Mixed Mood: The not so united states?
}

\section{Authors}

Gin S Malhi ${ }^{1,2,3,4^{*}}$, Michael Berk ${ }^{1,5,6}$, Grace Morris ${ }^{1,2,3,4}$, Amber Hamilton ${ }^{1,2,3,4}$, Tim Outhred ${ }^{1,2,3,4}$, Pritha Das $^{1,2,3,4}$, Darryl Bassett ${ }^{1,7}$, Bernhard T Baune ${ }^{1,8}$, Philip Boyce ${ }^{1,9}$, Bill Lyndon ${ }^{1,3,10,11}$, Roger Mulder ${ }^{1,12}$, Gordon Parker ${ }^{1,13,14}$, Ajeet B Singh ${ }^{1,15}$

${ }^{1}$ Mood Assessment and Classification Committee (MAC Committee). See Footnote.

${ }^{2}$ Academic Department of Psychiatry, Northern Sydney Local Health District, St Leonards, NSW,

Australia

${ }^{3}$ Sydney Medical School Northern, University of Sydney, Sydney, NSW, Australia

${ }^{4}$ CADE Clinic, Royal North Shore Hospital, Northern Sydney Local Health District, St Leonards, NSW, Australia

${ }^{5}$ Deakin University, School of Medicine, IMPACT Strategic Research Centre, Barwon Health, VIC, Australia

This is the author manuscript accepted for publication and has undergone full peer review but has not been through the copyediting, typesetting, pagination and proofreading process, which may lead to differences between this version and the Version of Record. Please cite this article as doi: $\underline{10.1111 / b d i .12502}$

This article is protected by copyright. All rights reserved 
${ }^{6}$ Department of Psychiatry, Orygen Research Centre, and the Florey Institute for Neuroscience and Mental Health, University of Melbourne, Melbourne, VIC, Australia

${ }^{7}$ Private Practice in Psychiatry and Division of Psychiatry, University of Western Australia

${ }^{8}$ Discipline of Psychiatry, University of Adelaide, Adelaide, South Australia, Australia

${ }^{9}$ Discipline of Psychiatry, Sydney Medical School, Westmead Clinical School, University of Sydney, Sydney, NSW, Australia

${ }^{10}$ Mood Disorders Unit, Northside Clinic, Greenwich, NSW, Australia

${ }^{11}$ ECT Services Northside Group Hospitals, Greenwich, NSW, Australia

${ }^{12}$ Department of Psychological Medicine, University of Otago - Christchurch, Christchurch, New Zealand

${ }^{13}$ School of Psychiatry, University of New South Wales

${ }^{14}$ Black Dog Institute, Sydney, New South Wales, Australia

${ }^{15}$ School of Medicine, IMPACT Strategic Research Centre, Deakin University, Geelong, Victoria, Australia

\section{*Correspondence}

MACC Chair. CADE Clinic, Academic Department, Level 3, Main Building, Royal North Shore Hospital, St Leonards, Sydney, NSW 2065, Australia.

Email: gin.malhi@sydney.edu.au

Footnote: The Mood Assessment and Classification Committee (MAC Committee) comprised academic psychiatrists with clinical expertise in the management of mood disorders and researchers with an interest in depression and bipolar disorders. The independently convened committee specifically targeted contentious aspects of mood disorders diagnosis and assessment with the express aim of informing clinical practice and future research. Members of the committee held one face to face meeting in Sydney (Australia) to discuss the issues in depth and agree upon outcomes. These were then developed further via email correspondence.

\section{Keywords}

This article is protected by copyright. All rights reserved 
Bipolar disorder, mixed states, mixed episode, mixed features

Current syndromal definitions of depression and mania - described as occurring in depressive and bipolar disorders, fail to adequately capture their myriad presentations; this has important ramifications for research and clinical practice. Symptom-based subtypes in the real world such as melancholia and atypical depression contribute to this clinical heterogeneity, as do the frequent comorbid occurrence of anxiety, trauma and personality disorders. Thus, mixed presentations - ostensibly an admixture of elevated and depressive features - are common, and remain a distinct challenge for those who wish to pristinely define bipolar disorders solely on the basis of depressive and manic phases, irrespective of severity.

The most recent DSM incarnation attempts to address this problem by allowing the addition of mixed symptoms as a specifier to current manic, hypomanic or depressive episodes in those with a mood disorder, setting the threshold at three or more symptoms being present concurrently from the opposite pole $^{1}$. This replaces the DSM-IV definition of a mixed episode that stipulated coterminous fulfilment of criteria for both a depressive and manic episode (with the exception of duration), which was judged as too stringent. But have the criteria become too relaxed?

The significance of this question is that it challenges the premise upon which the DSM bases mood disorder diagnoses. Specifically, DSM-5 continues to assign primacy to mood - adopting essentially a cross-sectional stance when appraising mood disorders ${ }^{1}$. In practice, diagnosis necessarily decides clinical management, but the perspective that DSM-5 provides largely disregards the influence of considering the longitudinal pattern of illness. In its current form, the definition proposed by DSM has only limited usefulness - for example, capturing a snapshot of mood syndromes as part of statistical and epidemiological studies. However, in practice, and especially in the United States, the DSM primarily serves as a template for clinical management decisions, including determining indications for specific pharmacological approaches ${ }^{2}$ and deciding on the governance and allocation of resources. Clinically, in all settings, the proper diagnosis of mood disorders requires careful formulation on the basis of detailed anamnesis that takes into account the history of the illness as much as its current signs and symptoms. DSM-5 lacks this deeper and richer perspective, a failure that makes its simplistic mood-based model fundamentally flawed.

The DSM-5 criteria for mixed states are a significant departure from its predecessor and, as such, cannot be approximated to DSM-IV criteria. This essentially prevents reanalysis of data sets using the new criteria and hence there are no rigorous treatment studies that have used DSM-5 criteria for classifying 
mixed states. In the meantime, the approach advocated by DSM-5 for describing mixed states may result in future over-diagnosis of mixed features and misdiagnosis of many of the mood permutations that occur in bipolar disorder. It is also unlikely to inform management or provide a useful foundation for research into biological substrates. This is because, by design, the mixed features specifier will capture a very heterogeneous group of manifestations and this cannot be coded as a 'mixed episode' per se - an option that has been lost in the transition from DSM-IV to DSM-5. It is therefore unclear to what extent, and indeed how accurately, such a specifier will be employed in clinical practice and how any 'data' that it generates can be collated and examined. But reverting to the DSM-IV definition of mixed episodes is equally unhelpful, as such a definition has yielded few insights since its formulation two decades ago. This is particularly problematic when tackling major depression, where the mixed states rubric implies a bipolar diathesis and usually prompts the adoption of a bipolar treatment paradigm - an approach that may in fact be ineffective in treating unipolar depressive symptoms with mixed features. Therefore we propose returning to a more systematic and nuanced definition of mixed states that will ensure that the central features of mixed states are fully appreciated and appropriately represented.

Descriptions of mixed states can be traced back to Weygandt and Kraepelin ${ }^{3}$. Their multi-axial approach attached equal emphasis to activity and thinking alongside mood, and in doing so they generated six mixed states in addition to the two 'pure forms' of mania and depression (see Figure 1). Re-

introducing these additional axes of activity and cognition into our taxonomy and practice could provide a much-needed sophisticated perspective for research and clinical care. It is likely that the various subtypes of mixed states will differ in prevalence, but detailed scrutiny of candidate states may allow distillation to a few. Naturally, increasing the number of 'kinds' of mixed states will create issues for researchers concerning statistical power, in particular the larger numbers of subjects needed for phenomenological and treatment studies. This contrasts with the approach taken by DSM-5 in which the definition of the mixed features specifier excludes certain symptoms on the basis of being overlapping and therefore supposedly non-discriminatory ${ }^{1}$. We believe that the consideration of whether overlapping symptoms distinguish mixed states from depression or mania is a separate exercise from that of defining and subtyping mixed states themselves. In particular, distractibility, irritability, psychomotor agitation and diminished ability to think or concentrate, or indecisiveness, need to be retained as specifiers, and the effects of treatment (iatrogenic mixed symptoms) also need to be included as a potential contributing factor in studies refining the symptoms that constitute mixed presentations. This is essential if we are to achieve a better understanding of mixed states processes, and until a clear picture emerges as to which symptoms and their inter-dependencies are clinically salient, multiple symptoms should be considered as potentially part of a mixed state.

This article is protected by copyright. All rights reserved 
Therefore our specific suggestions are two-fold. First - to consider activity and cognition over time as key dimensions in defining mixed states. Second - within the mixed features specifier of DSM-5, these symptoms need to be re-introduced as core components needing further study because mixed symptom complexes that include these features may need differential management (see Box 1).

Ultimately, refining the diagnosis of mixed mood states should lead to more effective management. Specifically, a dimensional approach to defining symptoms, along with greater consideration of aetiology and longitudinal course may allow clinicians to target therapies with precision. Mixed states are likely to be heterogeneous in origin, with some induced by treatment such as antidepressants, others related to personality or comorbidities, others part of the transition between high and low mood states, leaving a kernel of genuinely intrinsic mixed states. Treatment will therefore vary depending on these multiple factors and the course of illness, but will only be meaningfully possible if the diagnoses themselves are more sophisticated and specific in the first place. Thus a united approach, involving researchers and clinicians worldwide, is required to establish a consensus on the definition of mixed states; one that accurately reflects clinical reality.

\section{Acknowledgement}

The MAC Project was supported logistically by Servier who provided financial assistance with travel and accommodation for those MAC Committee members travelling interstate or overseas to attend the meeting in Sydney (held on $18^{\text {th }}$ March 2017). None of the committee were paid to participate in this project and Servier have not had any input into the content, format or outputs from this project.

\section{Disclosures}

The authors do not have any commercial associations that may pose a conflict of interest in connection with this manuscript.

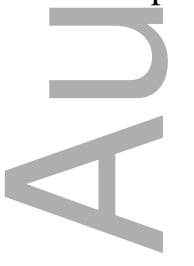

\section{References}


${ }^{1}$ American Psychiatric Association (APA) (2013). Diagnostic and Statistical Manual of Mental Disorders. $5^{\text {th }}$ edition. Washington DC: American Psychiatric Publishing. American Psychological Association.

${ }^{2}$ Malhi GS, Bassett D, Boyce P, Bryant R, Fitzgerald PB, Fritz K, Hopwood M, Lyndon B, Mulder R, Murray G, Porter R. Royal Australian and New Zealand College of Psychiatrists clinical practice guidelines for mood disorders. Australian \& New Zealand Journal of Psychiatry. 2015; 49(12): 1087-206.

${ }^{3}$ Marneros A. Origin and development of concepts of bipolar mixed states. Journal of affective disorders. 2001; 67(1): 229-40.

${ }^{4}$ Malhi GS, Byrow Y, Fritz K. Mixed Mood: time to adopt a 3D perspective? Australian \& New Zealand Journal of Psychiatry. 2016; 50(7): 613-615.

${ }^{5}$ Malhi GS, Byrow Y, Outhred T, Fritz K. Exclusion of overlapping symptoms in DSM-5 mixed features specifier: heuristic diagnostic and treatment implications. CNS Spectrums. 2016;1-8.

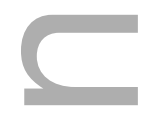

Figure 1. Eight faces of mood disorder illness perturbation. Variations in the activation $(+)$ and inhibition (-) of mood, thought and activity result in two pure states and six subtypes of mixed states, as initially conceptualised by Weygandt and Kraepelin. Pure Mania - activated mood, thought, and activity [Upper, Left face]. Pure Depression - inhibited mood, thought, and activity [Lower, Right face]. Depressive Mania - inhibited mood, and activated thought and activity [Lower, Left face]. Depressive Excitement $=$ inhibited mood and thought, and activated activity [Lower, Posterior face]. Depression FOI (with flight of ideas) - inhibited mood and activity, and activated thought [Lower, Anterior face]. Manic Stupor - activated mood, and inhibited thought and activity [Upper, Right face]. Inhibited Mania activated mood and thought, and inhibited activity [Upper, Anterior face]. Unproductive Mania activated mood and activity, and inhibited thought [Upper, Posterior face].

Box 1: Suggested mixed states sub-domains to enhance research and potentially better guide optimal clinical care. 


\section{University Library}

\section{- M M N E R VA A gateway to Melbourne's research publications}

Minerva Access is the Institutional Repository of The University of Melbourne

Author/s:

Malhi, GS;Berk, M;Morris, G;Hamilton, A;Outhred, T;Das, P;Bassett, D;Baune, BT;Boyce, P;Lyndon, B;Mulder, R;Parker, G;Singh, AB

Title:

Mixed mood: The not so united states?

Date:

2017-06-01

\section{Citation:}

Malhi, G. S., Berk, M., Morris, G., Hamilton, A., Outhred, T., Das, P., Bassett, D., Baune, B. T., Boyce, P., Lyndon, B., Mulder, R., Parker, G. \& Singh, A. B. (2017). Mixed mood: The not so united states?. BIPOLAR DISORDERS, 19 (4), pp.242-245. https://doi.org/10.1111/ bdi. 12502 .

Persistent Link:

http://hdl.handle.net/11343/292978 